00

\title{
Столкновения атомов лития в основном состоянии. Комплексные сечения спинового обмена
}

\author{
(C) B.A. Картошкин \\ Физико-технический институт им. А.Ф. Иофрфе РАН, \\ 194021 Санкт-Петербург, Россия \\ e-mail: victor.kart@mail.ioffe.ru
}

Поступила в редакцию 22.12.2020 г.

В окончательной редакции 22.12.2020 г.

Принята к публикации 11.01.2021 г.

Рассмотрено взаимодействие двух поляризованных атомов лития $\left({ }^{7} \mathrm{Li}\right)$. При столкновении таких атомов наряду с упругим рассеянием имеет место процесс спинового обмена, т. е. обмен электронной поляризацией между сталкивающимися атомами. Одновременно с переносом поляризации процесс спинового обмена приводит к сдвигу частоты магнитного резонанса сталкивающихся атомов. На основании данных по потенциалам взаимодействия между двумя атомами лития впервые проведен расчет энергетических зависимостей сечений спинового обмена и сдвига частоты магнитного резонанса.

Ключевые слова: спиновый обмен, поперечные сечения, сдвиги частоты.

DOI: 10.21883/OS.2021.05.50881.311-20

\section{Введение}

Димер лития - одна из наиболее изученных двухатомных молекул, причем как экспериментально, так и теоретически. Это связано с тем, что атом лития и соответственно димер лития являются наиболее простыми объектами исследований, если не принимать во внимание изотопы водорода. В связи с этим в литературе имеется достаточно большое количество работ, посвященных потенциалам взаимодействия димера $\mathrm{Li}_{2}$, как теоретических [1-3], так и основанных на данных, полученных в результате различных экспериментальных исследований [4-6].

В настоящей работе рассмотрено взаимодействие с участием двух атомов изотопа ${ }^{7} \mathrm{Li}$, находящихся в основном состоянии $1 s^{2} 2 s$. Рассматриваемые атомы лития в основном состоянии имеют нескомпенсированный электронный спин $S=1 / 2$, соответственно при столкновении двух атомов лития образуется димер с полным спином $S=1,0$.

В работе рассмотрено взаимодействие двух поляризованных атомов лития, находящихся в основном состоянии. В этом случае при столкновении атомов происходит обмен электронной поляризацией между сталкивающимися атомами (так называемый спиновый обмен) [7,8]. Таким образом, если перед столкновением один из атомов был поляризован, то он может передать имеющуюся у него поляризацию партнеру по столкновению [9]. Это позволяет осуществлять непрямую [10] поляризацию атомных частиц в случаях, когда осуществить прямую поляризацию (например, поляризованным оптическим излучением) оказывается невозможно. Подобный процесс характеризуется сечением спинового обмена $q_{\mathrm{se}}$. Вместе с передачей поляризации при спин-обменном столкновении имеет место также и сдвиг частоты маг- нитного резонанса поляризованных атомов [11]. Сдвиг частоты магнитного резонанса может быть описан с помощью сечения сдвига $q_{\mathrm{sh}}$.

В работе на основании данных по потенциалам взаимодействия двух атомов лития в основном состоянии рассчитаны комплексные сечения спинового обмена в интервале энергий столкновения от $2 \cdot 10^{-4}$ до $10^{-2}$ a.u.

\section{1. Потенциалы взаимодействия сталкивающихся атомов лития}

Как уже отмечалось выше, взаимодействие двух атомов лития $\left({ }^{7} \mathrm{Li}\right)$ исследуется достаточно давно. Это обусловлено тем, что атомы лития имеют самую простую (если не принимать во внимание атомы изотопов водорода) электронную структуру. Основное состояние атома ${ }^{7} \mathrm{Li}$ имеет электронную конфигурацию $1 s^{2} 2 s$. Таким образом, у атома лития в основном состоянии имеется электронный спин $S=1 / 2$. Также атом ${ }^{7} \mathrm{Li}$ имеет ядерный спин $I=3 / 2$. При столкновении двух атомов лития образуется молекула $\mathrm{Li}_{2}$. Поскольку время столкновения атомов (порядка $10^{-12} \mathrm{~s}$ ) существенно меньше времени сверхтонкого взаимодействия в атоме лития (порядка $10^{-9} \mathrm{~s}[12]$ ), то в процессе столкновения происходит взаимодействие только между электронами, a дальнейшее перераспределение поляризации между электронными и ядерными степенями свободы происходит между столкновениями за счет сверхтонкого взаимодействия. При столкновении атомов лития образуется димер с полным спином $S=1,0$. Эти состояния могут быть описаны с помощью двух потенциалов - триплетного, соответствующего полному спину $S=1\left(a^{3} \Sigma_{u}^{+}\right)$, и синглетного, соответствующего полному спину $S=0$ $\left(X^{1} \Sigma_{g}^{+}\right)$. 
Исследованию вышеприведенных молекулярных термов в литературе уделялось большое внимание. В частности, синглетный терм исследовался в работах $[2,3,6]$, а триплетный — в $[1,13,14]$.

В настоящей работе исследовано взаимодействие двух атомов изотопа ${ }^{7} \mathrm{Li}$, которое может быть описано с помощью синглетного и триплетного термов. При столкновении атомов друг с другом возможен процесс упругого рассеяния. Если сталкивающиеся атомы обладают нескомпенсированным электронным спином, то возможен также и процесс спинового обмена, т. е. обмен электронными степенями свободы между сталкивающимися атомами.

\section{1. Синглетный потенциал взаимодействия двух атомов лития в основном состоянии}

Для расчета интересующих нас комплексных сечений спинового обмена был использован синглетный потенциал, рассчитанный в работе [6]. В работе методом фурье-спектроскопии исследовались вращательные переходы в $A-X$-системе для атомов изотопа ${ }^{7} \mathrm{Li}$. На основании полученных экспериментальных данных были определены параметры синглетного потенциала взаимодействия в интервале межъядерных расстояний от $R_{\min }=2.516948 \AA$ (соответствующего колебательному уровню $v=0$, в атомных единицах длины $R_{\min }=4.756317$ a.u.) до $R_{\max }=12.492700$ до $\AA$ (соответствующего колебательному уровню $v=40$, в атомных единицах длины $R_{\max }=23.607657$ a.u.). Для проведения расчетов необходимо знать часть потенциала взаимодействия на межьядерных расстояниях меньше $R_{\min }$. Для расчета этой части потенциала воспользуемся методикой, предложенной, в частности, в [15]. Потенциальная энергия $V_{\mathrm{SR}}$ для межъядерных расстояний, меньших $R_{\min }=2.516948 \AA$, представляется обычно в виде $V_{\mathrm{SR}}=A+B / R^{N_{\mathrm{s}}}$. Подбор параметров $A, B$ и $N_{\mathrm{s}}$ производится таким образом, чтобы осуществить сшивку потенциальных кривых до и после $R_{\min }$. На рис. 1 представлен синглетный потенциал взаимодействия, построенный на основании табличных данных [6], с короткодействующей частью, рассчитанной в настоящей работе по методике, предложенной в [15]. На рисунке потенциал взаимодействия приведен в атомных единицах. Параметры короткодействующей части синглетного потенциала взаимодействия $\left(R \leq R_{\min }\right)$, представленной на рис. 1, имеют следующие значения (в обратных сантиметрах и ангстремах): $A=-12044.87509 \mathrm{~cm}^{-1}$, $B=80490.77484 \mathrm{~cm}^{-1} \AA^{2.27468}, N_{\mathrm{s}}=2.27468$.

Представленный синглетный потенциал характеризуется энергией диссоциации $D_{\mathrm{e}}=8516.61 \mathrm{~cm}^{-1}$ (в атомных единицах энергии $D_{\mathrm{e}}=0.03884$ a.u.) и равновесным расстоянием $R_{\mathrm{e}}=2.67299391 \AA$ (в атомных единицах длины $R_{\mathrm{e}}=5.0511199$ a.u.).

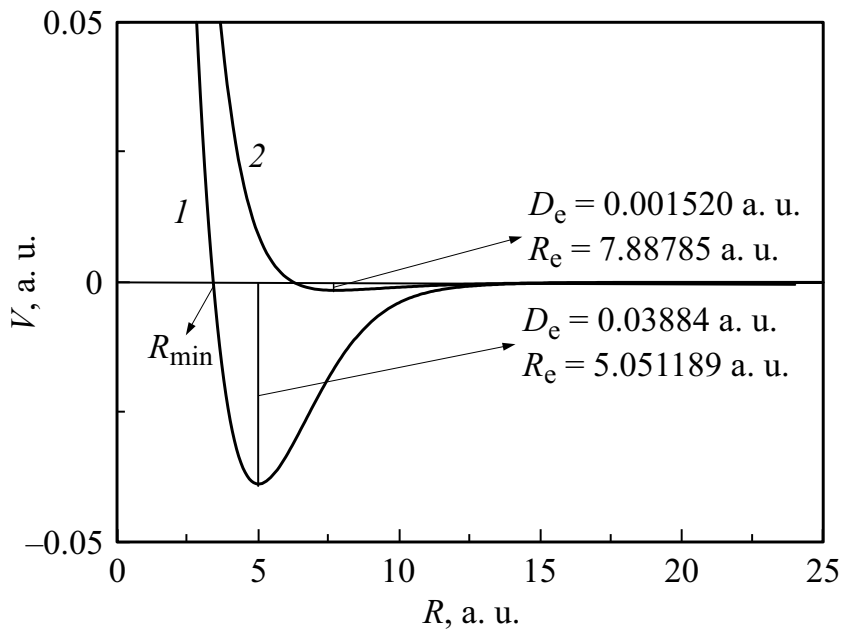

Рис. 1. Синглетный (1) [6] и триплетный (2) [1] потенциалы взаимодействия димера ${ }^{7} \mathrm{Li}-{ }^{7} \mathrm{Li}$ (атомная система единиц). Короткодействующая часть синглетного потенциала при $R \leq R_{\min }$ построена в настоящей работе. Для синглетного потенциала $D_{e}=8516.61 \mathrm{~cm}^{-1}$ (в атомной системе единиц $D_{e}=0.3884$ a.u.), $R_{e}=2.67299391 \AA$ (в атомной системе единиц $R_{e}=5.0511199$ а.u.); для триплетного терма $D_{e}=333.69 \mathrm{~cm}^{-1}\left(D_{e}=0.01520\right.$ a.u. $), R_{e}=0.4173 \mathrm{~nm}$ $\left(R_{e}=7.885785\right.$ a.u. $)$.

\section{2. Триплетный потенциал взаимодействия двух атомов лития в основном состоянии}

В настоящей работе для расчета сечений использовался триплетный потенциал взаимодействия, рассчитанный в работе [1]. Расчет данного потенциала проведен в интервале межъядерных расстояний от $R_{\min }=3.05$ a.u. до $R_{\max }=37.05$ a.u. Представленный триплетный потенциал характеризуется энергией диссоциации $\quad D_{\mathrm{e}}=333.69 \mathrm{~cm}^{-1} \quad\left(D_{\mathrm{e}}=0.001520\right.$ a.u. $)$ и равновесным расстоянием $R_{\mathrm{e}}=0.4173 \mathrm{~nm}$ $\left(R_{\mathrm{e}}=7.885785\right.$ a.u. $)$ [16]. На рис. 1 этот потенциал представлен в атомной системе единиц.

\section{2. Комплексные сечения спинового обмена}

Ниже приводится методика расчета комплексного сечения спинового обмена, которая аналогична представленной в $[7,8]$. Как известно, при столкновении двух атомных частиц, обладающих электронными спинами, кроме процесса упругого рассеяния, возможен также процесс обмена электронами, а если одна из частиц была предварительно поляризована, то и обмен электронной поляризацией. Подобный процесс условно представляется следующим образом:

$$
A(\uparrow)+B(\downarrow) \leftrightarrow A(\downarrow)+B(\uparrow) .
$$

Здесь стрелками показана возможная электронная поляризация частиц. 
Процесс спинового обмена можно описать с помощью комплексного сечения спинового обмена вида $[7,8]$ :

$$
q^{A B}=\bar{q}^{A B}+i \overline{\bar{q}}^{A B}
$$

Действительная часть комплексного сечения $\left(\bar{q}^{A B}\right)$ определяет перенос поляризации при столкновении частиц, релаксацию, образование высших поляризационных моментов (выстраивание, сверхтонкая поляризация). Мнимая часть сечения $\left(\overline{\bar{q}}^{A B}\right)$ определяет сдвиги частоты магнитного резонанса в системе как зеемановских, так и сверхтонких уровней атомов лития. Следовательно, зная комплексное сечение спинового обмена, можно полностью описать процессы, происходящие при спин-обменных столкновениях. Следует отметить, что сдвиги частоты магнитного резонанса существенно влияют на точностные характеристики приборов квантовой электроники, либо построенных с использованием явления спинового обмена, либо в случаях, когда спиновый обмен является ,паразитным процессом“ (квантовые магнитометры с оптической накачкой на щелочных атомах [17], квантовые стандарты частоты и времени [18] и т. д.)

Комплексное сечение спинового обмена можно представить стандартным образом через матрицу рассеяния [19]:

$$
q^{A B}=\frac{\pi}{k_{A B}^{2}}(2 l+1)\left[1-T_{0}^{A B}(l) T_{1}^{A B}(l)^{*}\right] .
$$

Здесь $k_{A B}^{2}=\mu_{A B} v_{A B} / \hbar-$ волновой вектор, $\mu_{A B}-$ приведенная масса сталкивающихся частиц $A$ и $B, v_{A B}-$ средняя относительная скорость сталкивающихся атомов, символ * указывает на комплексное сопряжение. Матрица рассеяния выражается через фазы рассеяния $\left(\delta_{S}^{A B}(l)\right)$ в канале с полным спином $S$ следующим образом:

$$
T_{S}^{A B}(l)=\exp \left(2 i \delta_{S}^{A B}(l)\right),
$$

где $l$ - орбитальное квантовое число.

Из выражений (3) и (4) следует, что действительная и мнимая части комплексного сечения имеют вид

$$
\begin{aligned}
& \bar{q}^{A B}=\frac{\pi}{k_{A B}^{2}} \sum_{l=0}^{\infty}(2 l+1) \sin ^{2}\left[\delta_{1}^{A B}(l)-\delta_{0}^{A B}(l)\right], \\
& \overline{\bar{q}}^{A B}=\frac{\pi}{k_{A B}^{2}} \sum_{l=0}^{\infty}(2 l+1) \sin ^{2}\left[\delta_{1}^{A B}(l)-\delta_{0}^{A B}(l)\right] .
\end{aligned}
$$

Таким образом, для расчета интересующих нас сечений необходимо рассчитать фазы рассеяния на синглетном и триплетном термах. Расчет фаз рассеяния проведен в квазиклассическом приближении в интервале энергий $10^{-4}-10^{-2}$ a.u., что соответствует интервалу температур от 30 до $3000 \mathrm{~K}$. Применение квазиклассического приближения обусловлено тем, что вклад в сечения вносит большое количество парциальных волн $l$ даже при низких энергиях столкновения.

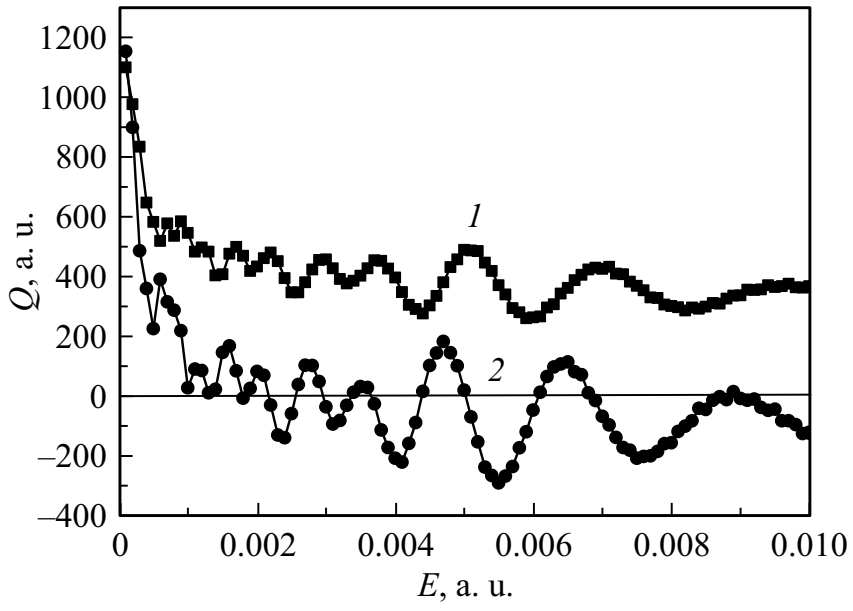

Рис. 2. Зависимость действительной (1) и мнимой (2) частей комплексного сечения спинового обмена при столкновении атомов ${ }^{7} \mathrm{Li}$ в основном состоянии от энергии столкновения.

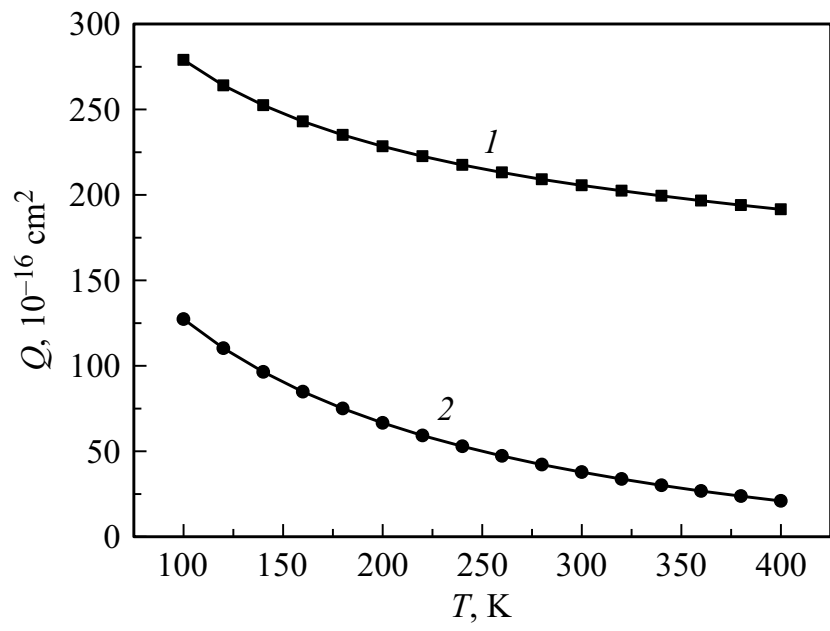

Рис. 3. Зависимость от температуры действительной (1) и мнимой (2) частей комплексного сечения спинового обмена при столкновении атомов ${ }^{7} \mathrm{Li}$ в основном состоянии.

На рис. 2 приведены полученные в результате расчета зависимости действительной и мнимой частей комплексного сечения спинового обмена для системы ${ }^{7} \mathrm{Li}-{ }^{7} \mathrm{Li}$. Как видно из рисунка, сечение сдвига $\overline{\bar{q}}^{A B}$ (кривая 2) в исследуемом интервале энергий стремится в сторону отрицательных значений, сечение обмена остается в области положительных значений. Для использования полученных в работе сечений при обработке экспериментальных данных необходимо перейти от энергетических к температурным зависимостям сечений. С этой целью необходимо было провести максвелловское усреднение сечений по скоростям. На рис. 3 представлены результаты такого усреднения. Из рисунка видно, что как сечение сдвига частоты, так и сечение спинового обмена положительны во всем интервале исследуемых температур. 


\section{Финансирование работы}

Работа выполнена в рамках госзадания, тема 00402019-0017.

\section{Конфликт интересов}

Автор заявляет, что у него нет конфликта интересов.

\section{Список литературы}

[1] Shi De-Heng, Sun Jin-Feng, Zhu Zun-Lue, Liu Yu-Fang // Chinese Phys. 2007. V. 16. N 9. P. 2701.

[2] Konowalowa D.D., Olson M.L. // J. Chem. Phys. 1979. V. 71. N 1. P. 450. doi 10.1063/1.438090

[3] Le Roy J., Dattani N.S., Coxon J.A., Ross A.J., Crozet P., Linton C. // J. Chem. Phys. 2009. V. 131. P. 204309. doi $10.1063 / 1.3264688$

[4] Wang X., Yang J., Qi J., Lyyra A.M. // J. Mol. Spectrosc. 1998. V. 191. N 2. P. 295.

[5] Wang X., Magnes J., Lyyra A.M., Ross A.J., Martin F., Dove P.M., Le Roy R.J. // J. Chem. Phys. 2002. V. 117. N 20. P. 9339. doi 10.1063/1.1514670

[6] Coxon J.A., Melville T.C. // J. Mol. Spectrosc. 2006. V. 235. N 2. P. 235. doi $10.1016 /$ jms.2005.11.009

[7] Картошкин В.А. // Опт. и спектр. 1998. Т. 85. № 1. С. 196; Kartoshkin V.A. // Opt. Spectrosc. 1998. V. 85. N 1. P. 177.

[8] Дмитриев С.П., Доватор Н.А., Картошкин В.А. // ЖТФ. 2015. T. 85. № 6. C. 40; Dmitriev S.P., Dovator N.A., Kartoshkin V.A. Technical Physics. 2015. V. 60. N 6. P. 826. doi 10.1134/S1063784215060079

[9] Картошкин В.А. // Опт. и спектр. 2010. Т. 109. № 5. С. 734; Kartoshkin V.A. // Opt. Spectrosc. 2010. V. 109. N 5. P. 674. doi 10.1134/S0030400X10110056

[10] Клементьев Г.В., Мельников В.Д., Картошкин В.А. // Химическая физика. 1985. Т. 4. № 1. С. 37.

[11] Картошкин В.А. // Опт. и спектр. 2010. Т. 108. № 6. С. 914; Kartoshkin V.A. // Opt. Spectrosc. 2010. V. 108. N 6. P. 866. doi 10.1134/S0030400X1006007X

[12] Puchalski M., Pachucki K. // Phys. Rev. Lett. 2013. V. 111. P. 243001. doi 10.1103/PhysRevLett.111.243001

[13] Linton C., Murphy T.L., Martin F., Bacis R., Verges J. // J. Chem. Phys. 1989. V. 91. N 10. P. 6036. doi 10.1063/1.457421

[14] Konowalow D.D., Regan R.M., Rosenkrantz M.E. // J. Chem. Phys. 1984. V. 81. N 10. P. 4534. doi 10.1063/1.447424

[15] Steinke M., Knöckel H., Tiemann E. // Phys. Rev. A. 2012. V. 85. P. 042720. doi 10.1103/PhysRevA.85.042720

[16] Linton C., Martin F.J., Ross A., Russier I., Crozet P., Yiannopoulou A., Li L., Lyyra A.M. // J. Mol. Spectrosc. 1999. V. 196. N 1. P. 20.

[17] Александров Е.Б., Вершовский А.К. // УФН. 2009. T. 179. № 6. C. 605-637; Aleksandrov E.B., Vershovsky A.K. // Phys. Usp. 2009. V. 52. N 6. P. 573. doi 10.3367/UFNe.0179.200906f.0605

[18] Affolderbach C., Droz F., Mileti G. // IEEE Trans. Instr. Measur. 2006. V. 55. N 2. P. 429. doi 10.1109/TIM.2006.870331

[19] Мотm Н., Месси Г. Теория атомных столкновений. М.: Мир, 1969. 756 с. 